\title{
Photoproduction of $\mathrm{H}_{3}^{+}$from gaseous methanol inside dense molecular clouds
}

\author{
S. Pilling ${ }^{1}$, D. P. P. Andrade ${ }^{2}$, A. C. F. Santos ${ }^{2}$, and \\ H. M. Boechat-Roberty ${ }^{2}$ \\ ${ }^{1}$ LNLS, Laboratório Nacional de Luz Síncrotron, São Paulo, Brazil. \\ email: sergiopilling@yahoo.com.br \\ ${ }^{2}$ UFRJ, Universidade Federal do Rio de Janeiro, Rio de Janeiro, Brazil. \\ email: diana_andrade@ufrj.br, toni@if.ufrj.br, heloisa@ov.ufrj.br
}

\begin{abstract}
We present experimental results obtained from photoionization and photodissociation processes of abundant interstellar methanol $\left(\mathrm{CH}_{3} \mathrm{OH}\right)$ as an alternative route for the production of $\mathrm{H}_{3}^{+}$in dense clouds. The measurements were taken at the Brazilian Synchrotron Light Laboratory (LNLS) employing soft X-ray and time-of-flight mass spectrometry. Mass spectra were obtained using the photoelectron-photoion coincidence techniques. Absolute averaged cross sections for the production of $\mathrm{H}_{3}^{+}$due to molecular dissociation of methanol by soft X-rays ( $\mathrm{C} 1 \mathrm{~s}$ edge) were determined. The $\mathrm{H}_{3}^{+}$'s photoproduction rate and column density were been estimated adopting a typical soft X-ray luminosity inside dense molecular and the observed column density of methanol. Assuming a steady state scenario, the highest column density value for the photoproduced $\mathrm{H}_{3}^{+}$was about $10^{11} \mathrm{~cm}^{2}$, which gives the ratio photoproduced/observed of about $0.05 \%$, as in the case of dense molecular cloud AFGL 2591. Despite the small value, this represent a new and alternative source of $\mathrm{H}_{3}^{+}$into dense molecular clouds and it is not been considered as yet in interstellar chemistry models.
\end{abstract}

Keywords. Methods: laboratory, molecular data, ISM: molecules, astrochemistry

\section{Introduction}

The $\mathrm{H}_{3}^{+}$ion plays an important role in diverse fields from chemistry to astronomy, for example in the chains of reaction that lead to the production of many of complex molecular species observed in the interstellar medium (Herbst \& Klemperer 1973, Dalgarno \& Black 1976, McCall et al. 1998 and references therein). A detailed review about this simplest stable interstellar polyatomic molecule could be found in Oka (2006).

In interstellar regions its main formation pathway occurs via ionization of molecular hydrogen by ubiquitous cosmic rays or local X-rays, followed by the efficient ion-neutral reaction, $\mathrm{H}_{2}^{+}+\mathrm{H}_{2} \rightarrow \mathrm{H}_{3}^{+}+\mathrm{H}$. Its dominant destruction pathway occurs via proton-hop reaction with the abundant interstellar carbon monoxide, $\mathrm{H}_{3}^{+}+\mathrm{CO} \rightarrow \mathrm{HCO}^{+}+\mathrm{H}_{2}$. However, as pointed out by Maloney et al. (1996) and Koyama et al. (1996), dense clouds, mainly the ones with embedded protostars, the soft X-ray field may represents the dominant excitation/ionization source, penetrating great depths into molecular clouds.

\section{Experimental methodology and results}

In an attempt to simulate the effect of stellar soft X-ray flux on gaseous molecules inside dense clouds we have used synchrotron radiation as a light source. The measurements were taken at the toroidal grating monochromator (TGM) beamline at Brazilian Synchrotron Light Laboratory (LNLS), Brazil, employing soft X-rays photons over the C1s resonance energy range $(200-310 \mathrm{eV})$. The incoming radiation perpendicularly intersects 
the gas sample inside a high vacuum chamber. Conventional time-of-flight mass spectra were obtained using photoelectron and photoion coincidence (PEPICO) techniques. The complete description of the experimental setup can be found elsewhere (Boechat-Roberty et al. 2005, Pilling et al. 2006, 2007b).

Methanol is one of the most abundant molecules in the interstellar medium and in dense molecular clouds. Therefore, even despite the reduced production of $\mathrm{H}_{3}^{+}$from Xrays photodissociation process, it is reasonable to expect that at least a fraction of the detected $\mathrm{H}_{3}^{+}$in molecular clouds may be produced from this simple methyl compound molecule.

The averaged cross sections for $\mathrm{H}_{3}^{+}$production by soft $\mathrm{X}$-rays photons at $\mathrm{C} 1$ s resonance, were determined taking into an account the relative intensities of the $\mathrm{H}_{3}^{+}$'s dissociative channels on PEPICOs spectra and the respective simple ionization and double ionization cross section of the parent molecule (see details in Pilling et al. 2007a).

The $\mathrm{H}_{3}^{+}$'s photoproduction rate and column density were estimated by adopting a typical soft X-ray luminosity inside dense molecular cloud (Stäuber et al. 2005) and the observed column density of its most abundant parent ion, methanol. The values for $\mathrm{H}_{3}^{+}$'s photoproduction cross section due to the dissociation of methanol by photons over the C1s edge were about $1.4 \times 10^{-18} \mathrm{~cm}^{2}$.

\section{Conclusion}

Assuming a steady state scenario and a typical X-ray luminosity of $L_{x} \gtrsim 10^{31} \mathrm{erg} \mathrm{s}^{-1}$ as the case for AFGL 2591 (Stäuber et al. 2005), the highest column density value for the photoproduced $\mathrm{H}_{3}^{+}$was about $10^{11} \mathrm{~cm}^{2}$, which gives the ratio photoproduced/observed of about $0.05 \%$. Despite the small value, this represents a new and alternative source of $\mathrm{H}_{3}^{+}$ inside dense molecular clouds that has not been considered as yet in interstellar chemistry models. Better estimative for $\mathrm{H}_{3}^{+}$photoproduction rate depends of more accurate soft $\mathrm{X}$-ray radiation field determinations.

Moreover, the energetic ionic products released by dissociation of $\mathrm{CH}_{3} \mathrm{X}$ molecules, including the $\mathrm{H}_{3}^{+}$ion, become an alternative and efficient route to complex molecular synthesis, since some ion-molecule reactions do not have an activation barrier and are also very exothermic. We hope that these cross sections give rise to more precise values for some molecular abundances in interstellar clouds and even in planetary atmosphere models.

\section{References}

Boechat-Roberty, H. M., Pilling, S., \& Santos, A. C. F. 2005, A\&A, 438, 915.

Dalgarno, A. \& Black, J. H. 1976, Rep. Prog. Phys., 39, 573.

Herbst, E., \& Klemperer, W. 1973, ApJ, 185, 505.

Koyama, K., Hamaguchi, K., Ueno, S., Kobayashi, N., \& Feigelson, E. D. 1996, PASJ (Letters), 48, L87.

Oka, T. 2006, PNAS, 103, 12235.

Maloney, P. R., Hollenbach, D. J., \& Tielens, A. G. G. M. 1996, ApJ, 466, 561.

McCall, B. J., Geballe, T. R., Hinkle, K. H., \& Oka, T. 1998, Science, 279, 1910.

Stäuber, P., Doty, S. D., van Dishoeck, E. F., \& Benz, A. O. 2005, A\&A A, 440, 949.

Pilling, S., Andrade, D. P. P., Neves, R., Ferreira-Rodrigues, A. M., Santos, A.C.F., \& BoechatRoberty, H. M. 2007a, MNRAS, 375, 1488.

Pilling, S., Neves, R., Santos, A. C. F., \& Boechat-Roberty, H. M. 2007b, Aש̇A, 464, 393.

Pilling, S., Santos, A. C. F., \& Boechat-Roberty, H. M. 2006, A\&A, 449, 1289. 